\title{
Optical Metrology Developments and Applications in the Transportation Industry
}

\author{
Michael Y. Y. Hung, FELLOW SPIE \\ City University of Hong Kong \\ Manufacturing Engineering and \\ Engineering Management \\ Department \\ Tat Chee Avenue, Kowloon \\ Hong Kong \\ E-mail: bcmhung@cityu.edu.hk \\ Frank Chen, MEMBER SPIE \\ Ford Motor Co. \\ Body/Chassis NVH and Squeak/Rattle \\ Prevention Dept. \\ Laser Imaging Lab. \\ and \\ Wayne State University \\ Detroit, Michigan 48202 \\ E-mail: fchen1@ford.com
}

Optical measurement techniques have enjoyed increasing popularity because of their advantages of being noncontacting, nonreinforcing, and generally full-field. Most of the techniques are imaging-based and hence are applicable to both very large objects and very small objects through proper adjustment of image magnification. In recent years, many developments and applications of optical techniques in the transportation industry have been reported. They include, to name a few, measurement of static and dynamic displacement, providing guidance for reduction of noise, vibration, and harshness (NVH); deformation measurement for strain/stress analysis, design verification, and optimization in terms of function and cost; shape or dimension gauging of components and full vehicle bodies, and manufacturing tools for quality improvement and cost reduction; quality inspection and nondestructive testing; as well as flow visualization, studies of combustion, aerodynamics, and heat transfer problems.

About five years ago, a special section on Optical Metrology: Developments and Applications in the Automotive Industry, was published in Optical Engineering. Since then, numerous new techniques, methods, and applications have been developed for the transportation sector. Today, it is felt that the subject should be updated. In this special section, the scope is enlarged to cover the entire transportation industry including automotive, aerospace, aircraft industries, and other related industries.

This special section includes a variety of examples ranging from new development and applications of opti- cal techniques in the aerospace and aircraft industry for nondestructive evaluation to micro/nanotechnology on microchip characterization. It consists of a collection of papers written by prominent researchers on holographic interferometry, electronic speckle pattern interferometry, digital holography, shearography, holographic moiré interferometry, structured light, laser Doppler velocimetry, and three-dimensional computer vision.

First in the section are two papers regarding recent shearography developments and applications. Kalms and Osten deal with large aircraft body and component defect detection using a mobile shearography system under industry environmental conditions by using mutual incoherent laser sources, while Hung et al. present a unified approach for holography and shearography in surface deformation measurement and various nondestructive testing applications including truck tire defect inspection.

Rastogi and Sharma provide a systematic approach of formula derivation in digital holography from which a concise and simple account of image formation is obtained with various recording configurations. Methods for recording large objects are reviewed and interferometric applications are discussed.

The following group of papers deals with developments and applications of micro/nanointerferometry with microscopes. Sciammarella and Sciammarella present techniques to measure objects with dimensions in the micro range using an electronic holographic moiré microscope and include an application example in the automo- 
tive industry. Developments to overcome issues with large magnifications and rigid body motion are discussed. Furlong and Pryputniewicz provide optoelectronic and microscopic techniques for characterization of shape and deformation of MEMS accelerometers with submicron accuracy. De Groot and de Lega develop a white light interference microscopy technique to measure geometric parameters of valves/cones that accommodate a wide range of cone angles and diameters without a change of optics. Experimental results show promising performance, including a $0.02-\mu \mathrm{m}$ standard deviation for cone roundness, including sample removal and replacement.

The next several papers are about shape and dimension measurement, which is a hot topic in the industry to aid in shortening development time and increasing product quality. Qian and Harding present an optimal approach to set up sensors to measure 3-D dimensions of an object by converting the signal dynamic range issues into distance problems in a spherical map. Then an algorithm is used to search near optimal orientations for sensor setup on the map. Ikeda et al. develop a frequency-modulated structured light technique for shape or dimension measurement, which is capable of providing absolute phase distribution and high-speed measurement. Measurements of object shape and deformation using the same setup are presented by Yang and Ettemeyer with electronic speckle pattern interferometry, while in the paper by Yamaguchi et al. it is achieved using digital holographic interferometry. Palmateer discusses the automated noncontact inspection of both small and large parts, which includes the process developed to assist in data collection and analysis.

Markov et al. develop a technique for structural integrity assessment of transportation systems by an optoacoustical sensor using a full-field laser Doppler velocimetry method. They show that the technique enables reliable defect detection, location, and quantification.

Chiang provides an overview on white light speckle pattern photography methods from the perspective of research works performed at SUNY. Special application examples in nano/micromechanics and heart mechanics are also presented. Helm et al. present the developments and applications of computer vision in fracture mechanics, in which 3-D shape and deformation are measured in conjunction with finite element analysis in wide, centernotched, and thin panels.

Rastogi and Sandoz explore an application of holographic moiré technique in the wood industry to measure and analyze the strength of finger-jointed wood assemblies, while Maranon et al. describe an application of a generic algorithm for quantitative characterization of a single delamination of composite laminated panels.

Shepard et al. present an active thermographic imaging technique to detect flaws and defects in automotive components using a simple one-dimensional model to reconstruct thermal signals that significantly reduces temporal noise, while Hung and Sheng present a 3-D computer vision method to measure the shapes and defects of objects with specularly reflective surfaces using reflectionstructured light techniques.

The last three papers concern using laser metrology in brake noise and vibration engineering. Stetson describes several optical metrology systems, including computerbased electronic holographic interferometry. The application in measuring the vibration mode of a brake rotor is also presented. Krupka et al. present a technique to measure the 3-D squeal mode of a squealing brake with 3-Dpulsed electronic speckle pattern interferometry. Application examples are also furnished. The last paper by Chen et al. provides various applications in brake noise and vibration engineering using various laser metrology methods.

It is hoped that this collection of papers will facilitate readers in understanding the state of the art of the subject and that it serves as a forum for exchanging ideas among researchers in this field.

Finally, we would like to thank all of the authors for their contributions and the reviewers for their valuable comments and efforts to ensure the high quality of the publications. We would also like to thank Dr. O'Shea, managing editor Ms. Labes, and the SPIE editing staff for providing both the opportunity and technical support for publishing this special section.

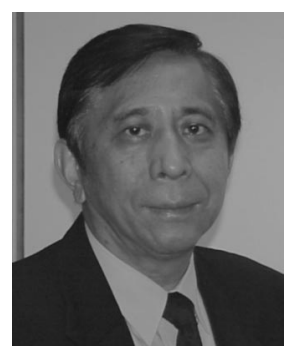

Michael Y. Y. Hung received his PhD in theoretical and applied mechanics from the University of Illinois. He is a chair professor in the Manufacturing Engineering and Engineering Management Department and a chair professor in the Building \& Construction Department at the City University of Hong Kong, Hong Kong. He also holds the John F. Dodge Professorship at Oakland University in USA. Prof. Hung has multi-disciplinary research experiences including: nondestructive testing, experimental stress analysis, 3-D computer vision, and optical metrology. He is a fellow of SEM and SPIE.

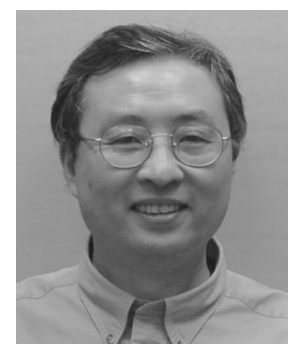

Frank Chen received his BS and MS degrees in engineering mechanics from Dalian University of Technology in China, in 1982 and 1985, respectively, and a $\mathrm{PhD}$ in mechanical system engineering from Oakland University, Michigan, in 1995. He was an assistant professor with Tongji University, Shanghai, China, from $1985-1989$. He is currently a technical specialist and group leader at laser imaging laboratory, Ford Motor Company, and an adjunct professor and PhD advisor at Wayne State University. He was the recipient of the Science and Technology Significant Contribution Award and Science and Technology Achievement Award from the National Education Committee of China in 1988 and 1989, respectively, and he received the Ford Customer Driven Quality Award, Ford Innovation Awards, Ford Truck Significant Achievement Award, and Ford Global Customer Satisfaction Award, in 1995, 1997, 1999, 1998, 2001, respectively. He has published 45 papers, coauthored 3 book chapters, and holds 5 patents. He has served as a co-chair of an international symposium in 2000 , an organization committee member of an international congress in 2002, and a co-guest editor for special sections in Optical Engineering in 1998, 2000, and 2003, respectively. His research interests concentrate on the developments and applications of various optical measurement techniques in noise, vibration, harshness $(\mathrm{NVH})$ reduction and dimension/shape measurement and control. 